\title{
Theoretical Limit of the Minimal Magnetization Switching Field and the Optimal Field Pulse for Stoner Particles
}

\author{
Z.Z. Sun and X. R. Wang \\ Physics Department, The Hong Kong University of Science and Technology, Clear Water Bay, Hong Kong SAR, China
}

(Received 7 May 2006; published 18 August 2006)

\begin{abstract}
The theoretical limit of the minimal magnetization switching field and the optimal field pulse design for uniaxial Stoner particles are investigated. Two results are obtained. One is the existence of a theoretical limit of the smallest magnetic field out of all possible designs. It is shown that the limit is proportional to the damping constant in the weak damping regime and approaches the Stoner-Wohlfarth (SW) limit at large damping. For a realistic damping constant, this limit is more than 10 times smaller than that of socalled precessional magnetization reversal under a noncollinear static field. The other is on the optimal field pulse design: if the magnitude of a magnetic field does not change, but its direction can vary during a reversal process, there is an optimal design that gives the shortest switching time. The switching time depends on the field magnitude, damping constant, and magnetic anisotropy.
\end{abstract}

Fabrication [1,2] and manipulation [3] of magnetic single-domain nanoparticles (also called the Stoner particles) are of great current interests in nanotechnology and nanosciences because of their importance in spintronics. Magnetization reversal, which is about how to switch a magnetization from one state to another, is an elementary operation. One important issue is how to switch a magnetization fast by using a small switching field. The switching field can be a laser light [4], or a spin-polarized electric current $[5,6]$, or a magnetic field [7,8]. Many reversal schemes $[9,10]$ have been proposed and examined. However, the issue of theoretical limits of the smallest switching field and the shortest switching time under all possible schemes are not known yet. Here we report two theorems on the magnetic-field induced magnetization reversal for uniaxial Stoner particles. One is about the theoretical limit of the smallest possible switching field. The other is about the optimal field pulse for the shortest switching time when the field magnitude is given.

Magnetization $\vec{M}=\vec{m} M$ of a Stoner particle can be conveniently described by a polar angle $\theta$ and an azimuthal angle $\phi$, shown in Fig. 1(a), because its magnitude $M$ does not change with time. The dynamics of magnetization unit direction $\vec{m}$ is governed by the dimensionless LandauLifshitz-Gilbert (LLG) equation $[3,8]$,

$$
\left(1+\alpha^{2}\right) \frac{d \vec{m}}{d t}=-\vec{m} \times \vec{h}_{t}-\alpha \vec{m} \times\left(\vec{m} \times \vec{h}_{t}\right),
$$

where $\alpha$ is a phenomenological damping constant whose typical value ranges from 0.01 to 0.22 for Co films [11]. The total field $\vec{h}_{t}=\vec{h}+\vec{h}_{i}$ comes from an applied field $\vec{h}$ and an internal field $\vec{h}_{i}=-\nabla_{\vec{m}} w(\vec{m})$ due to the magnetic anisotropic energy density $w(\vec{m})$. Different particle is characterized by different $w(\vec{m})$. In our analysis, we assume it uniaxial with the easy axis along the $z$ direction, $w=$ $w(\cos \theta)$ and $\vec{h}_{i}=-\frac{\partial w(\cos \theta)}{\partial(\cos \theta)} \hat{z} \equiv f(\cos \theta) \hat{z}$.
According to Eq. (1), each field generates two motions, a precession motion around the field and a damping motion toward the field as shown in Fig. 1(a). In terms of $\theta$ and $\phi$, Eq. (1) can be rewritten as [3]

$$
\begin{aligned}
\left(1+\alpha^{2}\right) \dot{\theta} & =h_{\phi}+\alpha h_{\theta}-\alpha f(\cos \theta) \sin \theta, \\
\left(1+\alpha^{2}\right) \sin \theta \dot{\phi} & =\alpha h_{\phi}-h_{\theta}+f(\cos \theta) \sin \theta .
\end{aligned}
$$

Here $h_{\theta}$ and $h_{\phi}$ are the field components along $\hat{e}_{\theta}-$ and $\hat{e}_{\phi}-$ directions of $\vec{m}$, respectively.

The switching problem is as follows: in the absence of an external field, the particle has two stable states, $\vec{m}_{0}$ (point $A$ ) and $-\vec{m}_{0}$ (point $B$ ) along its easy axis as shown in Fig. 1(b). Initially, the magnetization is $\vec{m}_{0}$, and the goal is to reverse it to $-\vec{m}_{0}$ by applying an external field. In our analysis, Gilbert damping constant $\alpha$ and the anisotropy $f(\cos \theta)$ are the fixed specifications of the problem, and only applied field variations are investigated. This is in contrast with earlier studies [12] where completely different analysis was performed. There are an infinite number of paths that connect the initial and the target state. L1 and
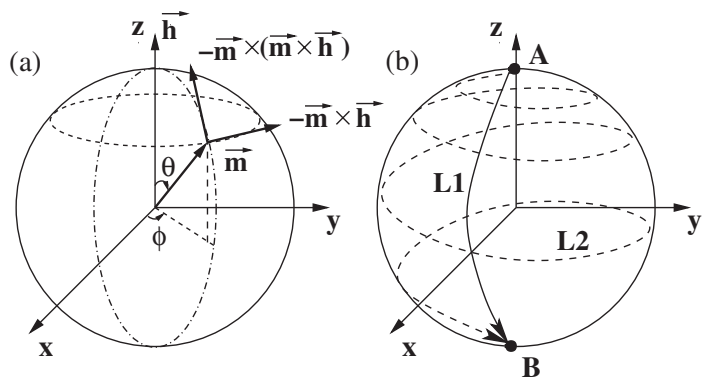

FIG. 1. (a) Two motions of magnetization $\vec{m}$ under field $\vec{h}$ : $-\vec{m} \times \vec{h}$ and $-\vec{m} \times(\vec{m} \times \vec{h})$ describe the precession and dissipation motions, respectively. (b) Points $A$ and $B$ represent the initial and the target states, respectively. The solid curve L1 and dashed curve L2 illustrate two possible reversal routes. 
L2 in Fig. 1(b) are two examples. Each of these paths can be used as a magnetization reversal route (path). Let $\vec{h}^{L, s}(t)$ be the magnetic field pulse of design $s$ along magnetization reversal route $L$. To proceed, a few quantities must first be introduced.

Definition of switching field $H^{L, s}$. - The switching field $H^{L, s}$ of design $s$ along route $L$ is defined to be the largest magnitude of $\vec{h}^{L, s}(t)$ for all $t, H^{L, s}=\max \left\{\left|\vec{h}^{L, s}(t)\right| ; \forall t\right\}$.

Definition of minimal switching field $H^{L}$ on reversal route $L$. - The minimal switching field along route $L$ is defined to be the smallest value of $H^{L, s}$ for all possible designs $s$ that will force the magnetization to move along $L$, i.e., $H^{L}=\min \left\{H^{L, s} ; \forall s\right\}$.

Definition of theoretical limit of minimal switching field $H_{c}$. - The switching field limit $H_{c}$ is defined as the smallest value of $H^{L}$ out of all possible routes, i.e., $H_{c}=$ $\min \left\{H^{L} ; \forall L\right\}$.

If the applied field is restricted to be static, reversal of a magnetization from $A$ to $B$ can only go through so-called "ringing motion" $[7,8]$. The corresponding switching field forms so-called modified Stoner-Wohlfarth (SW) astroid [7]. Strictly speaking, these switching fields are not $H^{L}$ that exists only for those ballistic reversal paths [8]. With the above remark about a static field, we come back to the first issue about $H_{c}$.

Theorem 1.-For a given uniaxial magnetic anisotropy specified by $f(\cos \theta)=-\frac{\partial w(\cos \theta)}{\partial(\cos \theta)}$, the theoretical limit of the minimal switching field is given by $H_{c}=\frac{\alpha}{\sqrt{1+\alpha^{2}}} Q$, where $Q=\max \{f(\cos \theta) \sin \theta\}, \theta \in[0, \pi]$.

Proof. - To find the lowest possible switching field, it should be noticed that field along the radius direction $h_{r}$ of an external field does not appear in Eq. (2). Thus one can lower the switching field by always putting $h_{r}=0$, and the magnitude of the external field is $h=\sqrt{h_{\theta}^{2}+h_{\phi}^{2}}$. According to Eq. (2), $\dot{\theta}$ and $\dot{\phi}$ are fully determined by $h_{\theta}$ and $h_{\phi}$ and vice versa. It can be shown that $h^{2}$ can be expressed in terms of $\theta, \phi, \dot{\theta}$, and $\dot{\phi}$

$$
\begin{aligned}
g \equiv & h^{2} \\
= & \left(1+\alpha^{2}\right) \dot{\theta}^{2}+2 \alpha f(\cos \theta) \sin \theta \dot{\theta}+(\alpha \sin \theta \dot{\phi})^{2} \\
& +\sin ^{2} \theta[\dot{\phi}-f(\cos \theta)]^{2} .
\end{aligned}
$$

Here $g(\dot{\theta}, \theta, \dot{\phi})$ does not depend explicitly on $\phi$ for a uniaxial model.

In order to find the minimum of $g$, it can be shown that $\phi$ must obey the following equation:

$$
\dot{\phi}=f(\cos \theta) /\left(1+\alpha^{2}\right)
$$

which is from $\left.\frac{\partial g}{\partial \phi}\right|_{(\dot{\theta}, \theta)}=0$ and $\left.\frac{\partial^{2} g}{\partial \dot{\phi}^{2}}\right|_{(\dot{\theta}, \theta)}>0$.

Equation (4) is a necessary condition for the smallest minimal switching field. This can be understood as follows. Assume $H_{c}$ is the minimal switching field along reversal path $L$ described by $\theta(t)=\theta_{1}(t)$ and $\phi(t)=$ $\phi_{1}(t)$ [i.e., $H_{c}$ is the maximum magnitude of the external field that generates the motion of $\theta_{1}(t)$ and $\left.\phi_{1}(t)\right]$. If $\phi_{1}(t)$ does not satisfy Eq. (4), then one can construct another reversal path $L^{*}$ specified by $\theta(t)=\theta_{1}(t)$ and $\phi(t)=$ $\phi_{2}(t)$, where $\phi_{2}(t)$ satisfies Eq. (4). Because $\theta(t)$ and $\dot{\theta}$ are exactly the same on both paths $L$ and $L^{*}$ at an arbitrary time $t$, the values of $g(t)$ shall be smaller on $L^{*}$ than those on $L$ at any $t$. Thus, the maximum $g^{*}=\left(H_{c}^{*}\right)^{2}$ of $g$ on $L^{*}$ will be also smaller than that $\left(H_{c}^{2}\right)$ on $L$, i.e., $H_{c}^{*}<H_{c}$. But this is in contradiction with the assumption that $H_{c}$ is the theoretical limit of the minimal switching field. Hence, $\phi(t)$ must obey Eq. (4) on the optimal path that generates the smallest switching field, $H_{c}$.

Substituting Eq. (4) into Eq. (3), we have

$$
h^{2}=\left[\sqrt{1+\alpha^{2}} \dot{\theta}+\alpha f(\cos \theta) \sin \theta / \sqrt{1+\alpha^{2}}\right]^{2} .
$$

In order to complete a magnetization reversal, the trajectory must pass through all values of $0 \leq \theta \leq \pi$. In particular, it must pass through whatever value of $\theta$ in that range maximizes $f(\cos \theta) \sin \theta$ on that range. At that maximizing value of $\theta$, the trajectory must be such that $\theta$ is nondecreasing, that is $\dot{\theta} \geq 0$, so that the trajectory is proceeding in the correct direction. Substituting these constraints into Eq. (5), we see that at that point in the trajectory, $h$ must be at least $\frac{\alpha Q}{\sqrt{1+\alpha^{2}}}$, where $Q \equiv \max \{f(\cos \theta) \sin \theta\}$. -QED.

To have a better picture about what this theoretical limit $H_{c}$ is, we consider a well-studied uniaxial model, $w(\vec{m})=$ $-k m_{z}^{2} / 2$, or $f=k \cos \theta$. It is easy to show that the largest $h$ is at $\theta=\pi / 4$ so that $Q=k / 2$, and

$$
H_{c}=\frac{\alpha}{\sqrt{1+\alpha^{2}}} \frac{k}{2} .
$$

At small damping, $H_{c}$ is proportional to the damping constant. The result in the limit of $\alpha \rightarrow 0$ coincides with the switching field in Ref. [9] where the time-dependent field always follows the motion of magnetization. At the large damping, $H_{c}$ approaches the SW field [8] when a noncollinear static switching field is $135^{\circ}$ from the easy axis. The solid curve in Fig. 2 is $H_{c}$ versus $\alpha$. For comparison, the minimal switching fields from other reversal schemes are also plotted. The dotted line is the minimal switching field when the applied field is always parallel to

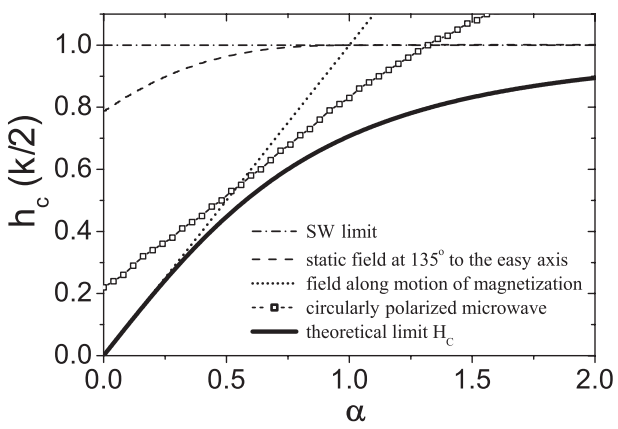

FIG. 2. The switching field $h_{c}$ vs damping constant $\alpha$ under different reversal schemes. 
the motion of the magnetization [9]. The curve in square symbols is the minimal switching field when a circularly polarized microwave at optimal frequencies is applied [9]. The dashed line is minimal switching field under a noncollinear static field of $135^{\circ}$ to the easy axis. It saturates to the SW field beyond $\alpha_{c}[7,8]$.

Although the theoretical limit of the switching field is academically important because it provides a low bound to the switching field so that one can use the theorem to evaluate the quality of one particular strategy, a design using a field at the theoretical limit would not be interesting from a practical point of view because the switching time would be infinitely long. Thus, it is more important to design a reversal path and a field pulse such that the reversal time is the shortest when the field magnitude $H$ $\left(H>H_{c}\right)$ is given. An exact result is given by the following theorem.

Theorem 2.-Suppose a field magnitude $H$ does not depend on time and $H>H_{c}$. The optimal reversal path (connects $\theta=0$ and $\theta=\pi$ ) that gives the shortest switching time is the magnetization trajectory generated by the following field pulse $\vec{h}(t)$,

$$
\begin{gathered}
h_{r}(t)=0, \quad h_{\theta}(t)=\alpha H / \sqrt{1+\alpha^{2}}, \\
h_{\phi}(t)=H / \sqrt{1+\alpha^{2}}=h_{\theta} / \alpha .
\end{gathered}
$$

Proof. - The reversal time from $A$ to $B$ [Fig. 1(b)] is $T \equiv$ $\int_{0}^{\pi} d \theta / \dot{\theta}$. According to Eq. (2), one needs $\left(h_{\phi}+\alpha h_{\theta}\right)$ to be as large as possible in order to make $\dot{\theta}$ maximal at an arbitrary $\theta$. Since $H^{2}=h_{r}^{2}+h_{\theta}^{2}+h_{\phi}^{2}$, one has the following identity:

$$
\begin{aligned}
\left(1+\alpha^{2}\right) H^{2}= & \left(1+\alpha^{2}\right) h_{r}^{2}+\left(h_{\phi}+\alpha h_{\theta}\right)^{2}+\left(h_{\theta}\right. \\
& \left.-\alpha h_{\phi}\right)^{2} .
\end{aligned}
$$

Thus, $\left(h_{\phi}+\alpha h_{\theta}\right)$ reaches the maximum of $\sqrt{1+\alpha^{2}} H$ when $h_{r}=0$ and $h_{\theta}=\alpha h_{\phi}$, which lead to Eq. (7), are satisfied.-QED

The optimal shape of field pulse (7) appears to depend only on the Gilbert damping constant $\alpha$ and not on $f(\cos \theta)$. However, those expressions provide the components of field magnitude in a coordinate system relative to the time-varying direction of $\vec{m}$. The magnetic anisotropy $f(\cos \theta)$ in part determines the trajectory of $\vec{m}$ which in turn determines the optimal pulse shape when combined with the expressions of Eq. (7). It should be pointed out that if they were to change $f(\cos \theta)$ and nothing else, the time-dependent field pulse would be different.

Under the optimal design of (7), $\phi(t)$ and $\theta(t)$ satisfy, respectively, Eq. (4) and

$$
\dot{\theta}=H / \sqrt{1+\alpha^{2}}-\alpha f(\cos \theta) \sin \theta /\left(1+\alpha^{2}\right) .
$$

For uniaxial magnetic anisotropy $w(\vec{m})=-k m_{z}^{2} / 2$, it is straightforward to integrate Eq. (9), and to find the reversal time $T$ from $A$ to $B$ [Fig. 1(b)],

$$
T=\frac{2}{k} \frac{\left(\alpha^{2}+1\right) \pi}{\sqrt{4\left(\alpha^{2}+1\right) H^{2} / k^{2}-\alpha^{2}}} .
$$

In the weak damping limit $\alpha \rightarrow 0, T \approx \pi / H$ while in the large damping limit $\alpha \rightarrow \infty, T \approx \frac{\alpha \pi}{\sqrt{H^{2}-k^{2} / 4}} \rightarrow \infty$. For the large field $H \rightarrow \infty, T \approx \frac{\sqrt{\alpha^{2}+1} \pi}{H}$, inversely proportional to the field strength. Thus, it is better to make $\alpha$ as small as possible. Then the critical field is low, and the speed is fast $(T \sim \pi / H)$. Figure 3 shows the field dependence of the switching time for $\alpha=0.1$, where $T$ and $H$ are in the units of $2 / k$ and $k / 2$, respectively.

How much could the so-called ballistic (precessional) reversal strategy $[7,13]$ be improved? To answer the question, let us compare the switching field and time in the ballistic reversal with those of theoretical limits for uniaxial magnetic anisotropy $w(\vec{m})=-k m_{z}^{2} / 2$ and $\alpha=0.1$. According to Ref. [8], the smallest switching field (in unit of $k / 2$ ) for the ballistic connection is $H=1.02$ applied in $97.7^{\circ}$ to the easy $z$ axis, and the corresponding ballistic reversal time (in unit of $2 / k$ ) is $T=5.87$. On the other hand, the theoretical limit for the minimal switching field is $H_{c} \approx 0.1$ from Eq. (6), about one tenth of the minimal switching field in the ballistic reversal [8]. For realistic value of $\alpha$ of order of 0.01 , the difference between experimentally achieved low switching field and the theoretical limit is of the order of hundred times. Thus there is a very large room for an improvement. It is also possible to switch a magnetization faster than that of the conventional ballistic reversal by using a smaller field. For example, to achieve a reversal time of $T=5.87$ along the optimal route, the field magnitude can be as lower as $H=0.547$ (instead of $H=1.02$ ) according to Fig. 3. To illustrate what scale of theoretical reversal is being demonstrated here, let us consider bulk fcc Co parameters of anisotropy constant $K=2.7 \times 10^{6} \mathrm{erg} / \mathrm{cm}^{3}$ and saturation magnetization $M_{s}=1445 \mathrm{emu} / \mathrm{cm}^{3}$ [14]. Thus the dimensionless reversal time of $T=5.87$ and switching field $H=0.547$ correspond to $178 \mathrm{ps}$ and $0.1 T$, respectively.

The field pulse given in Eq. (7) requires a constant adjustment of field direction during the magnetization

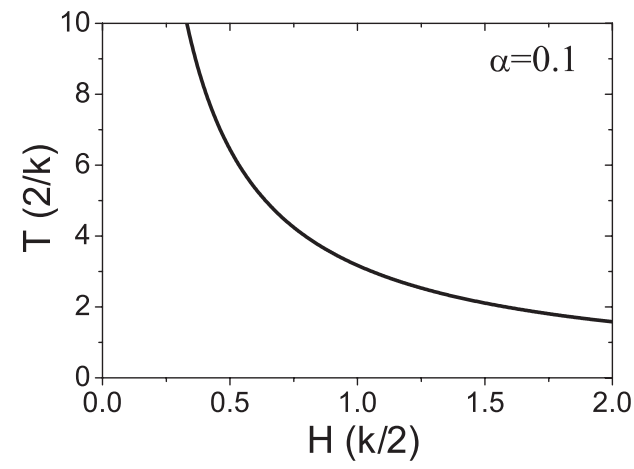

FIG. 3. The field dependence of $T$ under the optimal field pulse Eq. (7) for $\alpha=0.1$. The field is in the unit of $k / 2$ and the unit for time is $2 / k$. 

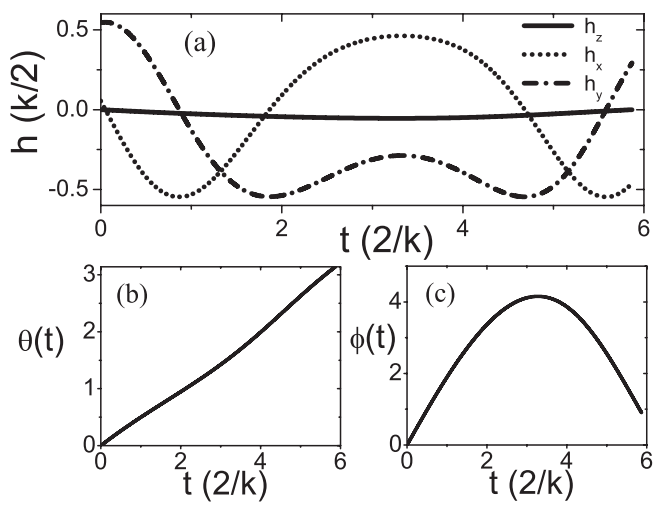

FIG. 4. Time dependence of different field components, $\theta$ and $\phi$, of $\vec{m}$ for uniaxial magnetic anisotropy $w(\vec{m})=-k m_{z}^{2} / 2$ with $\alpha=0.1$ and $H=0.547$ when the reversal path is optimal. The reversal time is $T=5.87$. (a) $x, y$, and $z$ components of magnetic field. (b) $\theta(t)$. (c) $\phi(t)$.

reversal. To have a better idea about the type of fields required, we plot in Fig. 4(a) the time dependence of $x$, $y$, and $z$ component of the field while its magnitude is kept at $H=0.547$. The time dependence of $\theta$ and $\phi$ is also plotted in Fig. 4(b) and 4(c).

Although the Stoner-Wohlfarth problem of magnetization reversal for a uniaxial model is of great relevance to the magnetic nanoparticles, it is interesting to generalize the results to the nonuniaxial cases. So far, our results are on the magnetic-field induced magnetization reversal; it will be extremely important to generalize the results to the spin-torque induced magnetization reversal. It should also be pointed out that it is an experimental challenge to create a time-dependent field pulse given by Eq. (7) in order to implement the optimal design reported here. This challenge could be met if a device sensitive to the motion of a magnetization can be found because a coil can be attached to the device to generate the required field. In principle, one may also use three mutually perpendicular coils to generate a given time-dependent field. This can be accomplished by controlling time-dependent electric currents through the coils.

In conclusion, the theoretical limit of the magnetization switching field for uniaxial Stoner particles is obtained. The limit is proportional to the damping constant at weak damping and approaches the SW field at large damping. When the field magnitude is kept to a constant, and the field direction is allowed to vary, the optimal field pulse and reversal time are obtained.

This work is supported by UGC, Hong Kong, through RGC CERG grants (No. 603106). A discussion with Professor J. Shi is acknowledged.
[1] Shouheng Sun, C. B. Murray, D. Weller, L. Folks, and A. Moser, Science 287, 1989 (2000); D. Zitoun, M. Respaud, M.-C. Fromen, M. J. Casanove, P. Lecante, C. Amiens, and B. Chaudret, Phys. Rev. Lett. 89, 037203 (2002).

[2] M. H. Pan, H. Liu, J. Z. Wang, J. F. Jia, Q. K. Xue, J. L. Li, S. Qin, U. M. Mirdaidov, X. R. Wang, J. T. Market, Z. Y. Zhang, and C. K. Shih, Nano Lett. 5, 87 (2005).

[3] Spin Dynamics in Confined Magnetic Structures I \& II, edited by B. Hillebrands and K. Ounadjela (SpringerVerlag, Berlin, 2001); J. Miltat, G. Albuquerque, and A. Thiaville, in Spin Dynamics in Confined Magnetic Structures I, edited by B. Hillebrands and K. Ounadjela (Springer-Verlag, Berlin, 2001).

[4] M. Vomir, L. H. F. Andrade, L. Guidoni, E. Beaurepaire, and J.-Y. Bigot, Phys. Rev. Lett. 94, 237601 (2005).

[5] J. Slonczewski, J. Magn. Magn. Mater. 159, L1 (1996); L. Berger, Phys. Rev. B 54, 9353 (1996).

[6] M. Tsoi et al., Phys. Rev. Lett. 80, 4281 (1998); E. B. Myers, D. C. Ralph, J. A. Katine, R. N. Louie, and R. A. Buhrman, Science 285, 867 (1999); J. A. Katine, F. J. Albert, R. A. Buhrman, E. B. Myers, and D.C. Ralph, Phys. Rev. Lett. 84, 3149 (2000); J. Z. Sun, Phys. Rev. B 62, 570 (2000); Z. Li and S. Zhang, Phys. Rev. B 68, 024404 (2003); Y.B. Bazaliy, B. A. Jones, and S.C. Zhang, Phys. Rev. B 57, R3213 (1998); 69, 094421 (2004).

[7] L. He and W. D. Doyle, IEEE Trans. Magn. 30, 4086 (1994); W. K. Hiebert, A. Stankiewicz, and M. R. Freeman, Phys. Rev. Lett. 79, 1134 (1997); T. M. Crawford, T. J. Silva, C. W. Teplin, and C. T. Rogers, Appl. Phys. Lett. 74, 3386 (1999); Y. Acremann, C. H. Back, M. Buess, O. Portmann, A. Vaterlaus, D. Pescia, and H. Melchior, Science 290, 492 (2000); C. Thirion, W. Wernsdorfer, and D. Mailly, Nat. Mater. 2, 524 (2003); D. Xiao, M. Tsoi, and Q. Niu, J. Appl. Phys. 99, 013903 (2006).

[8] Z.Z. Sun and X.R. Wang, Phys. Rev. B 71, 174430 (2005).

[9] Z.Z. Sun and X.R. Wang, Phys. Rev. B 73, 092416 (2006); cond-mat/0511135; cond-mat/0604013.

[10] W. Wetzels, G. E. W. Bauer, and O. N. Jouravlev, Phys. Rev. Lett. 96, 127203 (2006).

[11] C. H. Back, D. Weller, J. Heidmann, D. Mauri, D. Guarisco, E. L. Garwin, and H. C. Siegmann, Phys. Rev. Lett. 81, 3251 (1998); C. H. Back, R. Allenspach, W. Weber, S. S. P. Parkin, D. Weller, E. L. Garwin, and H. C. Siegmann, Science 285, 864 (1999).

[12] R. Kikuchi, J. Appl. Phys. 27, 1352 (1956).

[13] H. W. Schumacher, C. Chappert, P. Crozat, R. C. Sousa, P. P. Freitas, J. Miltat, J. Fassbender, and B. Hillebrands, Phys. Rev. Lett. 90, 017201 (2003); H. W. Schumacher, C. Chappert, R. C. Sousa, P. P. Freitas, and J. Miltat, Phys. Rev. Lett. 90, 017204 (2003).

[14] J.P. Chen, C. M. Sorensen, K. J. Klabunde, and G. C. Hadjipanayis, Phys. Rev. B 51, 11527 (1995). 